\title{
Information aggregation with continuum of types
}

Citation for published version (APA):

Bozbay, I., \& Peters, H. (2017). Information aggregation with continuum of types. Maastricht University, Graduate School of Business and Economics. GSBE Research Memoranda No. 032 https://doi.org/10.26481/umagsb.2017032

Document status and date:

Published: 12/12/2017

DOI:

10.26481/umagsb.2017032

Document Version:

Publisher's PDF, also known as Version of record

\section{Please check the document version of this publication:}

- A submitted manuscript is the version of the article upon submission and before peer-review. There can be important differences between the submitted version and the official published version of record.

People interested in the research are advised to contact the author for the final version of the publication, or visit the DOI to the publisher's website.

- The final author version and the galley proof are versions of the publication after peer review.

- The final published version features the final layout of the paper including the volume, issue and page numbers.

Link to publication

\footnotetext{
General rights rights.

- You may freely distribute the URL identifying the publication in the public portal. please follow below link for the End User Agreement:

www.umlib.nl/taverne-license

Take down policy

If you believe that this document breaches copyright please contact us at:

repository@maastrichtuniversity.nl

providing details and we will investigate your claim.
}

Copyright and moral rights for the publications made accessible in the public portal are retained by the authors and/or other copyright owners and it is a condition of accessing publications that users recognise and abide by the legal requirements associated with these

- Users may download and print one copy of any publication from the public portal for the purpose of private study or research.

- You may not further distribute the material or use it for any profit-making activity or commercial gain

If the publication is distributed under the terms of Article $25 \mathrm{fa}$ of the Dutch Copyright Act, indicated by the "Taverne" license above, 


\section{Maastricht University}

Irem Bozbay, Hans Peters

Information aggregation with continuum of types

$\mathrm{RM} / 17 / 032$

\section{GSBE}

Maastricht University School of Business and Economics

Graduate School of Business and Economics

P.O Box 616

NL- 6200 MD Maastricht

The Netherlands 


\title{
Information aggregation with continuum of types
}

\author{
İem Bozbay* $\quad$ Hans Peters ${ }^{\dagger}$
}

8th December 2017

\begin{abstract}
We consider an information aggregation problem where a group of voters wants to make a 'yes' or 'no' decision over a single issue. Voters have state-dependent common preferences, but hold possibly conflicting private information about the state in the form of types (signals). We assume that types are distributed from a state-dependent continuous distribution. In this model, Bayesian equilibrium voting and efficient voting coincide, and informative voting means that a voter votes in favor of the issue if and only if the signal exceeds a cut-point level. Our main result is an answer, in the form of a condition on the parameters of the model, to the question when informative voting is efficient.
\end{abstract}

Keywords: private information, efficient information aggregation, strategic voting.

JEL Classification Numbers: C70, D70, D71, D80, D82

\section{Introduction}

In many decision making situations, a group of people needs to accept or reject an issue. A policy is approved or rejected in a referendum; a defendant is convicted or acquitted by the jury in a court trial; a job candidate is hired or not by a hiring committee and so on. We focus on such collective decision making problems assuming that members of the committee share a common goal. Indeed, in both the jury and the hiring committee examples, it seems natural to assume that each member of the group agrees on the common goal, such as convicting the defendant if and only if she is guilty and hiring the candidate if and only if she is good at research. Similarly, in the referendum example, voters may all agree that approving the policy is good if and only if implementing it has a low cost. If any disagreements occur among the members, this may be caused by holding different kinds of evidence (about the defendant, the candidate or the costs of implementation of a new policy), or attaching different interpretations to the evidence.

In this paper, we model such situations as voting problems where individuals have state-dependent common preferences, but hold private information about what the state might be. One alternative is better than the other alternative for every voter under one

\footnotetext{
*University of Surrey, E-mail: i.bozbay@surrey.ac.uk

${ }^{\dagger}$ Maastricht University, E-mail: h.peters@maastrichtuniversity.nl
} 
state while the converse holds if the other state occurs. (We sometimes call this alternative the 'correct' alternative.) We express private information or evidence by voters' types (i.e., signals) and we specifically focus on the problem of designing a voting rule. A desirable property of a voting rule in this setting is that it should lead to 'truthful' voting behavior, in the sense of following the evidence. A voting rule that generates this behavior allows for correct information aggregation, as the votes reveal the private information of individuals. Since all members of the group aim for reaching the better alternative, the voting rule should ideally lead to the outcomes that are most likely correct, given the available information; hence, aggregate private information efficiently. In this paper, we want to find out when voting rules exist which make truthful voting behavior efficient or, equivalently here, a Bayesian equilibrium.

The work on binary collective choice problems with common goals and private information started with the seminal works by Austen-Smith and Banks (1996) and Feddersen and Pesendorfer (1997). In this framework, they show that it need not be rational for all voters to vote truthfully. Taking this into account, Feddersen and Pesendorfer (1997) analyse asymptotic efficiency while Austen-Smith and Banks (1996) derive the conditions under which truthful voting occurs in equilibrium. The private information of a voter takes a binary value in these works. Duggan and Martinelli (2001) and Meirowitz (2002) extend these models by considering continuous rather than binary private information. They characterize the equilibrium of the game under fixed mechanisms and prove a jury theorem where the probability of a mistaken decision goes to zero as the size of the jury increases (with the exception of unanimity rule). One may think of several situations where private information of a voter cannot be summarized by a discrete variable or as indicating only 'yes' or 'no'. The evidence regarding the job candidate's skills in teaching may include the student evaluations, which take values in a given interval. The belief of a juror about a defendant may be less conclusive than 'guilty' or 'innocent'. Such information may be private or partly private: members of the hiring committee or jurors in a court of law may have access to different sources of information or may interpret the available evidence differently. In this paper, we assume that the private information of a voter is drawn from a continuous distribution and we aim to find whether there exist voting rules which lead to truthful behavior as well as efficient outcomes. We generally mean 'informative voting' by truthful behavior. In case of binary types, informativeness is easily defined as revealing the type information in the vote. In our paper, an informative voter who holds a type above a given cut-point accepts the issue, Similarly, an informative voter holding a type below the cut-point rejects the issue. ${ }^{1}$ Our model is similar to that of Duggan and Martinelli (2001) but we focus on mechanism design and any size of committees instead of equilibrium analysis under a fixed mechanism and large committees. Our paper can also be seen as an extension of Austen-Smith and Banks (1996) to a continuum of types.

It turns out that once we move from binary types to a continuum of types, we depart from previous results. We show that a voting rule which makes informative voting efficient exists under a specific condition on the model parameters. This result is in contrast with the one in Bozbay, Dietrich and Peters (2014), where there always exists a voting rule

\footnotetext{
${ }^{1}$ In our model, the higher values of a type indicate a higher likelihood that accepting the issue is the correct decision. Similarly, the lower values indicate a higher likelihood that rejecting the issue is the correct decision.
} 
efficiently aggregating private information when types are binary. ${ }^{2}$ The condition under which such a rule exists in our model (see Theorem 2) implies that there exists a threshold such that whenever an issue is more likely to be correct than not given all the available information, it should be more likely to be correct based on the information about the number of types exceeding this threshold. When the condition holds, i.e., this threshold exists, the informative strategy must be defined with this threshold as the cut-point and a quota rule must be used to make informative voting efficient. Hence, when private information reflects a richer set of possibilities rather than indicating 'yes' or 'no' only, a voting rule cannot always aggregate the whole available information efficiently. ${ }^{3}$

There are other related contributions focusing on information aggregation. In a recent work, Barelli, Bhattacharya, and Siga (2017) analyze asymptotic efficiency in a setting where there are at least two alternatives, voters have common state-dependent preferences but hold private signals which do not necessarily come from a set identical to the state space. Using majority rule as the mechanism, they show that there exists a sequence of equilibria that efficiently aggregates information as the group size goes to infinity only when the signal space is rich enough relative to the state space. Although we share in common that the signal and the state spaces do not coincide (and this plays an important role in both results), we depart from this work by considering a binary issue, and taking a mechanism design approach for small committees instead of focusing on information aggregation in large elections under a fixed mechanism. There is another strand of literature focusing on mechanism design for collective choice problems with two alternatives. Some of these works assume private values such as Azrieli and Kim (2014), who show that a rule is interim incentive efficient if and only if it is a weighted majority rule. Schmitz and Tröger (2012) consider a model in which the voters' utility profile is drawn from a joint distribution, and each voter's utility is private knowledge and expresses her preference between two alternatives; a choice rule assigns to each reported profile the probabilities with which the final alternative is chosen. In our model each voter can assess the probability of each state given her private information, and these probabilities play the role of the utilities in the Schmitz-Tröger model. Hence, we focus on a much more detailed version of their model, and therefore are able to express our results on the basis of these details, namely the objective probabilities of the states and the signal functions, as well as the binary voting method.

Truthful behavior might alternatively be represented by 'sincere voting', which means voting without taking other voters into consideration. The classical Condorcet jury theorem framework does not distinguish between informative and sincere behavior; moreover, assumes that voters always vote informatively. Austen-Smith and Banks (1996) show that this assumption is inconsistent with a game-theoretic view of collective behavior. As the equivalence of sincere behavior and informative behavior cannot be taken for granted, in Section 3 we determine the condition under which a sincere voter votes informatively (see Theorem 1). This condition determines the particular informative strategy defined by a cut-point which should balance the role of prior beliefs. Section 4 focuses on the

\footnotetext{
${ }^{2}$ They mainly analyse multi-issue voting, where a voter receives a 'yes' or 'no' signal for each issue. The one issue model appears as a special case of their model.

${ }^{3}$ Of course, a basic assumption of our model is that voters can only vote 'yes' or 'no' for each issue, but this is also a realistic assumption in most applications, like the ones mentioned above.
} 
key question of efficient information aggregation. Section 5 concludes. All proofs are in Appendix.

\section{The Model}

We consider a group of voters, labelled $i=1, \ldots, n$, where $n \geq 2$. This group needs to collectively accept or reject an issue. This issue may represent a referendum, a policy, or a judgment on whether a defendant in a court is guilty. A vote takes the form 1 or 0 which means 'accept/yes' or 'reject/no' respectively. After all voters cast their votes, a collective decision in $\{0,1\}$ is taken using a voting rule. Formally, a voting rule is a function $g:\{0,1\}^{n} \rightarrow\{0,1\}$, mapping each voting profile $\mathbf{v}=\left(v_{1}, \ldots, v_{n}\right)$ to a decision $d \equiv g(\mathbf{v})$.

We denote by $s$ the state of the world. We assume that there are two possible states of the world $s_{0}$ and $s_{1}$, so $s \in\left\{s_{0}, s_{1}\right\}$. By assumption, the prior probability that the state is $s_{1}$ is denoted

$$
\pi=\operatorname{Pr}\left(s_{1}\right)
$$

and belongs to $(0,1)$. Thus, the prior probability that the state is $s_{0}$ is $1-\pi$.

Voters have identical preferences, captured by a common utility function $u:\{0,1\} \times$ $\left\{s_{0}, s_{1}\right\} \rightarrow \mathbb{R}$ which maps any decision-state pair $(d, s)$ to its utility $u(d, s)$. The utility function is given by

$$
u(d, s)= \begin{cases}1 & \text { if } d=s_{d} \\ 0 & \text { if } d \neq s_{d}\end{cases}
$$

So, the utility is state-dependent: voters commonly prefer 1 if the state is $s_{1}$ and they commonly prefer 0 if the state is $s_{0}$.

Each voter has a type, representing private information or evidence. A voter's type is denoted by $t \in[0,1]$. The type could represent information that is not shared with other voters because of a lack of deliberation or limits of deliberation. More generally, a voter $i$ 's type could represent uncertainty of other voters about $i$ 's beliefs. When we refer to the type of a particular voter $i$, we write $t_{i}$. For each $i, t_{i}$ is distributed randomly according to the state conditional density $f_{1}$ or $f_{0}$ depending on whether the state of the world is $s_{1}$ or $s_{0}$. For instance, the probability that $t \leq \tau$ if the state is $s_{1}$, is given by the expression $\int_{0}^{\tau} f_{1}(t) d t$. We write $\mathbf{t}=\left(t_{1}, \ldots, t_{n}\right) \in[0,1]^{n}$ for a vector of voters' types. We denote by $T$ the random variable generating voters' types and by $S$ the random variable generating the state $s$. When we refer to the particular voter $i$, we write $T_{i}$. We make the following assumptions.

- Each type is independently drawn given the true state of the world. Formally, $T_{1}, \ldots, T_{n}$ are conditionally independent given $S$.

- The state conditional densities $f_{1}$ and $f_{0}$ are piecewise continuous and $f_{1}(t), f_{0}(t)>0$ for all $t \in[0,1]$.

- $\frac{f_{0}}{f_{1}}$ is weakly decreasing on $[0,1]$. 
The independence assumption allows us to characterize the joint distribution of the state and types. The second assumption implies that densities have common support. The last assumption is commonly used especially in mechanism design problems and is called the monotone likelihood ratio property. It implies that the distribution of types conditional on ' $s_{1}$ ' first-order stochastically dominates the distribution of types conditional on ' $s_{0}$ '. Formally, the conditional cumulative distribution function $F_{1}$ has first-order stochastic dominance over the conditional cumulative distribution function $F_{0}$; hence, $F_{0}(t) \geq F_{1}(t)$ for all $t \in[0,1]$. This assumption ensures that types convey information about the state: the higher values of types are stronger indications of the state being $s_{1}$. After a voter receives her type, she submits a vote in $\{0,1\}$. A voting strategy is a function $\sigma:[0,1] \rightarrow$ $\{0,1\}$, mapping each possible type $t \in[0,1]$ to that type's vote $\sigma(t)=v$. A strategy profile is a vector $\boldsymbol{\sigma}=\left(\sigma_{1}, \ldots ., \sigma_{n}\right)$ of strategies across voters.

With a voting rule $g$ and a common utility function $u$, we now have a well-defined Bayesian game. For a type profile $\mathbf{t}$, we call a decision $d \in\{0,1\}$ efficient if it has maximal expected utility conditional on the full information $\mathbf{t}$, i.e., if it maximises $E(u(d, S) \mid \mathbf{t})$. Some common notions of voting behavior can now be adapted to our framework:

- A strategy $\sigma$ of a voter is informative if there exists a cut-point $\hat{t} \in(0,1)$ such that $\sigma(t)=1$ if $t \geqslant \hat{t}$ and $\sigma(t)=0$ if $t<\hat{t}$ for all $t \in[0,1]$. This strategy is informative in the sense that it reveals information about the relation between a voter's type and the cut-point.

- A strategy $\sigma$ of a voter is sincere if for every type $t$, the vote $\sigma(t)$ maximizes the expected utility conditional on the information $t$, i.e., $d=\sigma(t)$ maximizes $E(u(d, S) \mid t)$. A sincere voter acts as if her vote alone determined the decision, which amounts to optimal behavior in a hypothetical single-player decision problem.

- A strategy profile $\boldsymbol{\sigma}=\left(\sigma_{1}, \ldots, \sigma_{n}\right)$ is an equilibrium if each strategy is a best response to the other strategies, i.e., if the profile is a Nash equilibrium of the corresponding Bayesian game.

In the definition of an informative strategy, in order to avoid technicalities, we have excluded the (anyway uninteresting) cases $\hat{t} \in\{0,1\}$. In particular, given the positivity of the signal density functions, we have $F_{s}(t)=0$ if and only if $t=0$ and $F_{s}(t)=1$ if and only if $t=1$, hence, at any cut-point $\hat{t}$, we have $0<F_{s}(\hat{t})<1$. We denote by $\sigma^{\hat{t}}$ the informative strategy with cut-point $\hat{t}$. The corresponding informative strategy profile, where each voter votes informatively according to the same cut-point $\hat{t}$, is denoted by $\boldsymbol{\sigma}^{\hat{t}}=\left(\sigma_{1}^{\hat{t}}, \ldots, \sigma_{n}^{\hat{t}}\right)$. Below, when considering informative voting, we always (and only) consider the case where all voters use the same cut-point. This is a natural assumption, given that all voters are completely symmetric apart from the realized signals. Since efficient voting and equilibrium voting in our model coincide - see Section 4 - this means that we only consider symmetric equilibria. Also, to avoid distraction by special cases, throughout we exclude parameter combinations such that the probability of more than one efficient decision is positive. In other words, we assume that for almost every type profile there is exactly one efficient decision. Finally, our restriction to informative voting is natural in the following sense: if the voting rule is a quota rule, which means that 
it chooses 1 if an only if the number of 1 -votes exceeds a given threshold, then we may assume that any best reply of a voter is informative - see Section 6.3 for the details.

\section{When is informative voting sincere?}

In the classical Condorcet Jury Theorem framework, it is generally assumed that informative voting is sincere; that is, voters are assumed to simply follow the (binary) type in their votes. Austen-Smith and Banks (1996) show that when voters are strategic, this cannot be taken for granted and holds only under some conditions. We now give a characterization of the parameter combinations where informative voting and sincere voting coincide in our setting. The proof of this theorem and later theorems can be found in the Appendix.

Theorem 1 The informative voting strategy with cut-point $\hat{t} \in(0,1)$ is sincere if and only if

(i) $\frac{\pi}{1-\pi} \leq \frac{f_{0}(\hat{t})}{f_{1}(\hat{t})}$ for all $t<\hat{t}$, and

(ii) $\frac{\pi}{1-\pi} \geq \frac{f_{0}(\hat{t})}{f_{1}(\hat{t})}$ for all for all $t>\hat{t}$.

Theorem 1 says that an informative strategy is sincere if the cut-point is chosen such that the ratio of priors is smaller (greater) than the likelihood ratio for the types that are smaller (greater) than the cut-point. It implies that a sincere informative voting strategy exists if and only if $\frac{\pi}{1-\pi} \in\left(\frac{f_{0}(1)}{f_{1}(1)}, \frac{f_{0}(0)}{f_{1}(0)}\right){ }^{4}$ If $\frac{f_{0}}{f_{1}}$ is continuous, this cut-point satisfies $\frac{\pi}{1-\pi}=\frac{f_{0}(\hat{t})}{f_{1}(\hat{t})}$. There may be an interval of cut-points making the associated informative strategy sincere. If $\frac{f_{0}}{f_{1}}$ is strictly decreasing, then there is a unique sincere informative strategy.

\section{When is informative voting efficient?}

Our objective is to find out whether a voting rule exists which makes informative voting behavior occur in equilibrium and moreover leads to efficient decisions. Recall that by saying 'informative voting' without referring to a particular voter, we mean informative voting by all voters according to a common cut-point. Whenever we say that informative voting is efficient, we mean this voting behavior leads to efficient decisions. Following a well-known result by McLennan (1998), it is sufficient to find out whether there exist voting rules which lead to efficient decisions under informative voting, as this also guarantees informative voting in equilibrium. In the case where private information takes a binary 'yes' or 'no' form, there always exist voting rules which efficiently aggregate private information (see Austen-Smith and Banks, 1996). The next theorem shows that efficient information aggregation is not always possible when voters' private information reflects a

\footnotetext{
${ }^{4}$ This is a consequence of our assumption that $\hat{t} \neq 0,1$. Without this assumption, we have that a voter would always choose 0 if $\frac{\pi}{1-\pi} \leq \frac{f_{0}(1)}{f_{1}(1)}$ and 1 if $\frac{\pi}{1-\pi} \geq \frac{f_{0}(0)}{f_{1}(0)}$.
} 
rich spectrum of possibilities instead of only indicating truth or falsity, and characterizes all parameter combinations for which efficient rules exist. To state our result, we define a notation first: For each type profile $\mathbf{t}$ and cut-point $\hat{t}$, we denote $n^{\mathbf{t}}=\left|\left\{i: t_{i} \geq \hat{t}\right\}\right|$.

Theorem 2 There exists a voting rule $g$ which makes informative voting with cut-point $\hat{t}$ efficient if and only if $\hat{t}$ satisfies

$$
\frac{\pi}{1-\pi}>\prod_{i \in\{1, \ldots, n\}} \frac{f_{0}\left(t_{i}\right)}{f_{1}\left(t_{i}\right)} \Longleftrightarrow \frac{\pi}{1-\pi}>\left(\frac{1-F_{0}(\hat{t})}{1-F_{1}(\hat{t})}\right)^{n^{\mathrm{t}}}\left(\frac{F_{0}(\hat{t})}{F_{1}(\hat{t})}\right)^{n-n^{\mathrm{t}}}
$$

for each type profile $\mathbf{t} .^{5}$

The implication (2) says that a state is more likely to be the true state given all the available information if and only if it is more likely to be the true state based on the information about the number of types exceeding the cut-point. In other words, the only information that should be relevant for information aggregation is the number of types exceeding the cut-point. Under (2) we can focus on whether each type is above or below the cut-point, and omit the differences in value.

The proof of Theorem 2 also hints at the kind of rules that make informative voting efficient, namely quota rules. A quota rule is defined by a threshold $m \in\{0,1, \ldots, n+1\}$ : for each voting profile it accepts the issue (hence, leads to $d=1$ ) if and only if at least $m$ voters accept it. In order to state the next theorem, we define the coefficient $m^{*}$ as follows. Consider all parameter combinations for which there exists a $\hat{t}$ satisfying (2), and for $\hat{t}$ define

$$
K=\left\{k \in\{0, \ldots, n\}: \frac{\pi}{1-\pi}>\left(\frac{1-F_{0}(\hat{t})}{1-F_{1}(\hat{t})}\right)^{k}\left(\frac{F_{0}(\hat{t})}{F_{1}(\hat{t})}\right)^{n-k}\right\}
$$

and

$$
m^{*}=\left\{\begin{array}{cc}
\min K & \text { if } K \neq \emptyset \\
n+1 & \text { if } K=\emptyset
\end{array}\right.
$$

This coefficient has a simple interpretation: for $s_{1}$ to be more probably the true state than not, there must be at least $m^{*}$ individuals whose types exceed the cut-point $\hat{t}$ in the type profile.

Theorem 3 If there exists a voting rule which makes informative voting with cut-point $\hat{t}$ efficient, then it is the quota rule with the threshold $m^{*}$.

As mentioned before, in Section 6.3 we show that under a quota rule it is without loss of generality to assume that any best reply of a voter is informative. In particular, this implies that under a quota rule, if decisions are efficient - hence every voter plays a best reply - then we may assume that voters vote informatively.

We now consider some examples. We start with a combination of parameters where (2) is satisfied, and a quota rule makes informative voting efficient. We then introduce

\footnotetext{
${ }^{5}$ Note that the denominators at the right-hand side are unequal to zero: see Section 3.
} 
another example where (2) fails. Under this combination of parameters, we illustrate the 'best' rule, which minimizes the probability that the efficient decision is not chosen. As these examples indicate, condition (2) is only satisfied in exceptional cases. The reader is referred to Section 6.1 for computations on which these examples are based.

Example 1 Let $n=2$ and $\pi=0.6$. The density functions are

$$
f_{0}(t)= \begin{cases}\frac{3}{2} & \text { if } 0<t<\frac{1}{2} \\ \frac{1}{2} & \text { if } \frac{1}{2} \leq t \leq 1\end{cases}
$$

and

$$
f_{1}(t)= \begin{cases}\frac{1}{2} & \text { if } 0<t<\frac{1}{2} \\ \frac{3}{2} & \text { if } \frac{1}{2} \leq t \leq 1\end{cases}
$$

The likelihood ratio is

$$
\frac{f_{0}(t)}{f_{1}(t)}= \begin{cases}3 & \text { if } 0<t<\frac{1}{2} \\ \frac{1}{3} & \text { if } \frac{1}{2} \leq t \leq 1\end{cases}
$$

and the corresponding cumulative distribution functions are

$$
F_{0}(t)= \begin{cases}\frac{3}{2} t & \text { if } 0<t<\frac{1}{2} \\ \frac{1}{2}+\frac{1}{2} t & \text { if } \frac{1}{2} \leq t \leq 1\end{cases}
$$

and

$$
F_{1}(t)= \begin{cases}\frac{1}{2} t & \text { if } 0<t<\frac{1}{2} \\ \frac{3}{2} t-\frac{1}{2} & \text { if } \frac{1}{2} \leq t \leq 1 .\end{cases}
$$

One can easily check that (2) is satisfied for $\hat{t}=\frac{1}{2}$. By Theorem 3 , the rule that makes informative voting efficient is the quota rule with the threshold $m=1$. Let us see this graphically. Figure 1 illustrates the types space for two voters, where each axis shows the type of each voter. The grey region shows all type profiles for which the efficient decision is $d=1$ while the white region shows those for which the efficient decision is $d=0$. Moreover, the quota rule with $m=1$ under informative voting with $\hat{t}=\frac{1}{2}$ leads to 1 for all the type profiles in the grey area (and to 0 in the white area).

This combination of parameters leads to an equivalent of the binary type model. Given informative voting with cut-point $\hat{t}=\frac{1}{2}$, a voter will vote 1 with probability $1-F_{1}\left(\frac{1}{2}\right)=\frac{3}{4}$ if the true state is 1 , and 0 with probability $F_{0}\left(\frac{1}{2}\right)=\frac{3}{4}$ if the true state is 0 . Think of a model with binary signals where a voter receives signal 1 with probability $\frac{3}{4}$ if the true state is 1 and signal 0 with probability $\frac{3}{4}$ if the true state is 0 . If this voter votes informatively, i.e., in accordance with the signal, and if the quota rule with $m=1$ is used, then this results in the same efficient decisions as the continuous type model. Indeed, in the model of Austen-Smith and Banks (1996), each voter receives a 'guilty' or 'innocent' signal. In our example with continuous signals, the 'guilty' signal is represented by all types in $[1 / 2,1]$ and the 'innocent' signal is represented by all types in $[0,1 / 2)$. 
a)

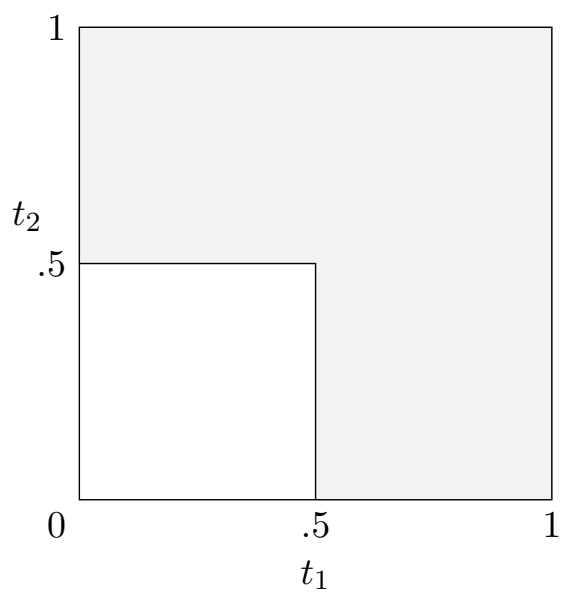

b)

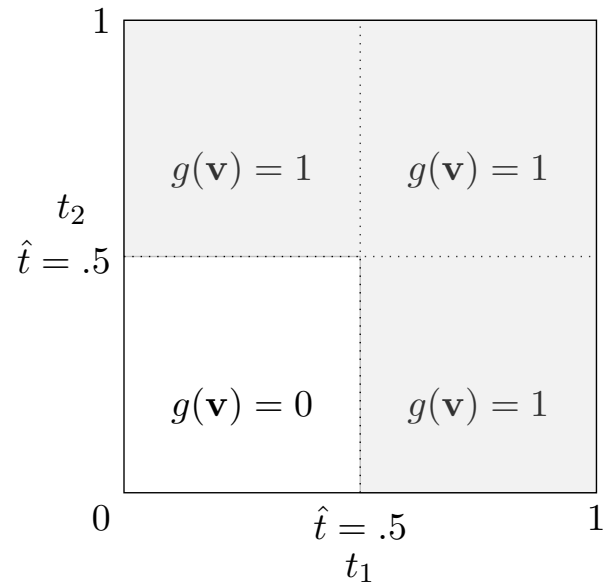

Figure 1: In a), the grey region shows all type profiles for which $d=1$ is the efficient decision while the white region shows those for which $d=0$ is the efficient decision. In $\mathrm{b}$ ), the regions illustrate the corresponding decisions under informative voting with cut-point $\hat{t}=0.5$ when $g$ is the quota rule with $m=1$.

Example 2 Let $n=2$ and $\pi=0.6028$. Suppose the density functions are

$$
f_{0}(t)=\frac{e^{1-t}}{e-1} \text { and } f_{1}(t)=1
$$

for each $t \in[0,1]$. The likelihood ratio is then $\frac{f_{0}(t)}{f_{1}(t)}=\frac{e^{1-t}}{e-1}$ for each $t \in[0,1]$. One can easily see that under this combination of parameters there exists no $\hat{t} \in(0,1)$ for which $(2)$ holds. Although we cannot always reach efficiency according to Theorem 2, can we specify a voting rule and a cut-point which lead to efficient decisions most often? In Figure 2a below, the area in grey (white) shows all type profiles for which $d=1(d=0)$ is efficient. Suppose the cut-point $\hat{t}$ is chosen as in Figure $2 \mathrm{~b}$, and consider the quota rule with $m=1$. This rule leads to the 'wrong' decision - the decision that is not efficient given the type profile - under informative voting whenever the type profile falls in the dotted area. If we instead choose $m=2$, the type profiles which lead to the inefficient decision under informative voting fall in the dotted area given in Figure 2c. 
a)

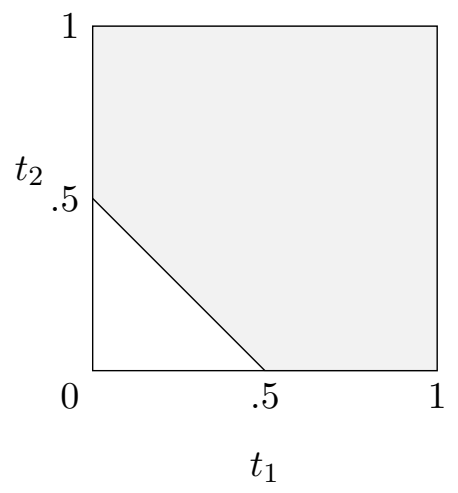

b)

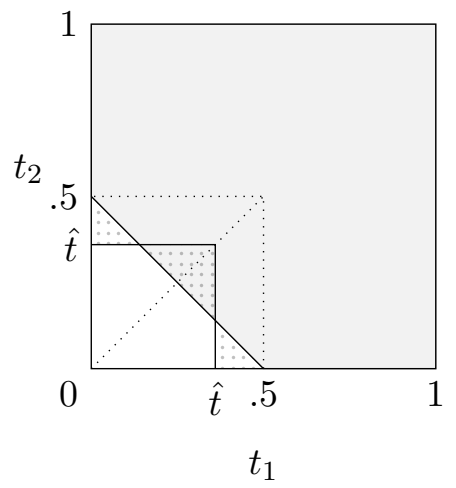

c)

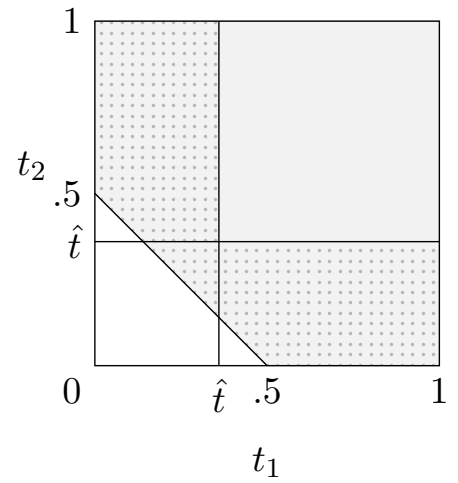

Figure 2: In a), the grey region shows all type profiles for which $d=1$ is the efficient decision while the white region shows those for which $d=0$ is the efficient decision. The dotted areas in b) and c) show those type profiles for which informative voting with a quota rule is not efficient, for $m=1$ and $m=2$ respectively.

It is easy to see that the probability of a wrong (inefficient) decision is much less in a setting such as in Figure 2b. We now calculate the probability that a given type profile $\mathbf{t}=\left(t_{1}, t_{2}\right)$ belongs to the dotted area in Figure $2 \mathrm{~b}$, assuming that the threshold $\hat{t}$ takes a value as shown in the figure. This probability is given by

$$
\begin{aligned}
& \int_{\frac{1}{2}-\hat{t} \frac{1}{2}-t_{1}}^{\hat{t}} \int_{\hat{t}}^{\hat{t}}\left(\pi f_{1}\left(t_{1}\right) f_{1}\left(t_{2}\right)+(1-\pi) f_{0}\left(t_{1}\right) f_{0}\left(t_{2}\right)\right) d t_{2} d t_{1} \\
& +2 \int_{\hat{t}}^{\frac{1}{2}} \int_{0}^{\frac{1}{2}-t_{1}}\left(\pi f_{1}\left(t_{1}\right) f_{1}\left(t_{2}\right)+(1-\pi) f_{0}\left(t_{1}\right) f_{0}\left(t_{2}\right)\right) d t_{2} d t_{1} .
\end{aligned}
$$

This probability is minimal for $\hat{t}=0.3031$. In other words, informative voting with this cut-point is most efficient. Similarly as in Example 1, the equivalent model with binary signals is the one where a voter receives signal 1 with probability 0.6969 (namely, $1-\hat{t}$ ) if the true state is 1 and signal 0 with probability 0.417 (namely, $F_{0}(\hat{t})$ ) if the true state is 0 . If this voter votes informatively, i.e., in accordance with the signal, and if the quota rule with $m=1$ is used, then this results in efficient decisions conditional on the binary signals.

\section{Conclusion}

We have considered a model where a group of voters with common interests wants to accept or reject an issue, where desirability of each outcome depends on the realization of the state of the world. Departing from the related literature where each voter's type 
takes the binary form, we have assumed that voters' types are distributed from a statedependent continuous distribution, thus allowing for a more realistic model. In contrast to the previous results (where informative voting is always and only efficient with quota rules), efficient information aggregation is possible only under a restrictive condition. This condition leads to the continuous analogue of the binary type model, which carries no additional information compared to the binary type model. Hence, it is not possible to efficiently aggregate continuum of types such as to reflect a richer spectrum of possibilities than in the binary types model, by a voting rule in which votes are binary.

In the perfectly aligned preferences setting, a natural question that arises is why voters do not share their private information as this would eventually lead to the implementation of the efficient decision. Austen-Smith and Feddersen (2006) analyze the effect of a prevoting deliberation in the case of binary signals when there is some degree of heterogeneity in preferences. Full information sharing occurs when the committee is not too diverse. Whether a deliberation stage under some heterogeneity in voter preferences may provide different intuitions in our model depends on how we define deliberation. Deliberation in the binary signal model can occur in the form of a yes/no straw poll before the actual election (as, for instance, in Coughlan 2000). Introducing such stage with binary actions would not improve the result in our setting. When types come from a continuous distribution, communicating them is not straightforward; hence, the assumption of limited deliberation, we believe, is well suited here.

\section{Appendix: proofs}

\subsection{Preliminary derivations}

Voter $i$ 's belief about the state of the world conditional on her type $t$ is represented by the expressions $\operatorname{Pr}\left(S=s_{1} \mid T=t\right.$ ) (and $\operatorname{Pr}\left(S=s_{0} \mid T=t\right)$ ). We abuse notation for simplicity and we write $\operatorname{Pr}\left(s_{1} \mid t\right)$. This expression can be determined as follows:

$$
\begin{aligned}
\operatorname{Pr}\left(s_{1} \mid t\right) & =\lim _{\Delta x \rightarrow 0} \operatorname{Pr}\left(s_{1} \mid t<T \leqslant t+\Delta x\right) \\
& =\lim _{\Delta x \rightarrow 0} \frac{\operatorname{Pr}\left(s_{1}, t<T \leqslant t+\Delta x\right)}{\operatorname{Pr}(t<T \leqslant t+\Delta x)} \\
& =\lim _{\Delta x \rightarrow 0} \frac{\pi f_{1}(t) \Delta x}{\left(\pi f_{1}\left(t_{1}\right)+(1-\pi) f_{0}(t)\right) \Delta x} \\
& =\frac{\pi f_{1}(t)}{\pi f_{1}(t)+(1-\pi) f_{0}(t)} .
\end{aligned}
$$

We can derive a similar expression for $\operatorname{Pr}\left(s_{0} \mid t\right)$, which gives

$$
\operatorname{Pr}\left(s_{0} \mid t\right)=\frac{(1-\pi) f_{0}(t)}{\pi f_{1}(t)+(1-\pi) f_{0}(t)}
$$

These expressions state the probability of a state $s \in\left\{s_{0}, s_{1}\right\}$ conditional on a voter's type $t \in[0,1]$. The probabilities of the states conditional on the full information $\mathbf{t} \in[0,1]^{n}$ can 
be derived similarly:

$$
\begin{aligned}
& \operatorname{Pr}\left(s_{1} \mid \mathbf{t}\right)=\frac{\pi \prod_{i \in\{1, \ldots, n\}} f_{1}\left(t_{i}\right)}{\prod_{i \in\{1, \ldots, n\}}\left(\pi f_{1}\left(t_{i}\right)+(1-\pi) f_{0}\left(t_{i}\right)\right)} \\
& \operatorname{Pr}\left(s_{0} \mid \mathbf{t}\right)=\frac{(1-\pi) \prod_{i \in\{1, \ldots, n\}} f_{0}\left(t_{i}\right)}{\prod_{i \in\{1, \ldots, n\}}\left(\pi f_{1}\left(t_{i}\right)+(1-\pi) f_{0}\left(t_{i}\right)\right)} .
\end{aligned}
$$

\subsection{Proofs}

Proof of Theorem 1. First, let us assume that $\sigma^{\hat{t}}$ is sincere for some some cut-point $\hat{t} \in(0,1)$. Then, for any given type $t \in[0,1], E(u(d, S) \mid t)$, and equivalently $\operatorname{Pr}(d \mid t)$ is maximal at $d=\sigma^{\hat{t}}(t)$. (By maximal, we mean $\operatorname{Pr}(d \mid t) \geq \operatorname{Pr}\left(d^{\prime} \mid t\right)$ for $d^{\prime} \neq d$.) Let $t \geq \hat{t}$, so $\sigma^{\hat{t}}(t)=1$. From $\operatorname{Pr}\left(s_{1} \mid t\right) \geq \operatorname{Pr}\left(s_{0} \mid t\right)$, we have $\frac{\pi}{1-\pi} \geq \frac{f_{0}(t)}{f_{1}(t)}$ by (5) and (6). Now, let $t<\hat{t}$; hence, $\operatorname{Pr}(d \mid t)$ is maximal at $d=0$. From $\operatorname{Pr}\left(s_{0} \mid t\right) \geq \operatorname{Pr}\left(s_{1} \mid t\right)$, we have $\frac{\pi}{1-\pi} \leq \frac{f_{0}(t)}{f_{1}(t)}$ by $(5)$ and (6).

Conversely, suppose $\hat{t} \in(0,1)$ satisfies conditions (i) and (ii) of the theorem. We want to show that $\sigma^{\hat{t}}$ is sincere; hence, that $\operatorname{Pr}(d \mid t)$ is maximal at $d=\sigma^{\hat{t}}(t)$ for any given type $t \in[0,1]$.

Let $t<\hat{t}$. This means $\sigma^{\hat{t}}(t)=0$. By (i), $\frac{\pi}{1-\pi} \leq \frac{f_{0}(t)}{f_{1}(t)}$ and $\operatorname{Pr}(d=0 \mid t)$ is maximal by (5) and (6). Similarly, let $t \geq \hat{t}$, so $\sigma^{\hat{t}}(t)=1$. By (ii), $\frac{\pi}{1-\pi} \geq \frac{f_{0}(t)}{f_{1}(t)}$. So, $\operatorname{Pr}(d=1 \mid t)$ is maximal (5) and (6).

Proof of Theorem 2. For parts (a) and (b) below, suppose a voting rule $g$ makes informative voting with some cut-point $\hat{t} \in(0,1)$ efficient. We wish to show that the following holds for every t:

$$
\frac{\pi}{1-\pi}>\prod_{i \in\{1, \ldots, n\}} \frac{f_{0}\left(t_{i}\right)}{f_{1}\left(t_{i}\right)} \Longleftrightarrow \frac{\pi}{1-\pi}>\left(\frac{1-F_{0}(\hat{t})}{1-F_{1}(\hat{t})}\right)^{n^{\mathrm{t}}}\left(\frac{F_{0}(\hat{t})}{F_{1}(\hat{t})}\right)^{n-n^{\mathrm{t}}} .
$$

(a) First, consider a given type profile $\mathbf{t}$ and suppose that a decision $d^{*}$ is efficient for t. This means

$$
\operatorname{Pr}\left(d^{*} \mid \mathbf{t}\right)>\operatorname{Pr}(d \mid \mathbf{t})
$$

for $d \neq d^{*}$. (Here we use that efficiency ties occur with probability zero and can be neglected.) Let $N^{\mathbf{t}}:=\left\{i: t_{i} \geq \hat{t}\right\}$. For all type profiles $\mathbf{t}^{\prime}$ with $\boldsymbol{\sigma}^{\hat{t}}\left(\mathbf{t}^{\prime}\right)=\boldsymbol{\sigma}^{\hat{t}}(\mathbf{t})$, we have $t_{i} \geq \hat{t} \Longleftrightarrow t_{i}^{\prime} \geq \hat{t}$, or equivalently $N^{\mathbf{t}}=N^{\mathbf{t}^{\prime}}$. Moreover, $d^{*}=g\left(\boldsymbol{\sigma}^{\hat{t}}(\mathbf{t})\right)=g\left(\boldsymbol{\sigma}^{\hat{t}}\left(\mathbf{t}^{\prime}\right)\right)$ is the common efficient decision for all such profiles. Hence, we can write

$$
\operatorname{Pr}\left(d^{*} \mid N^{\mathbf{t}^{\prime}}\right)>\operatorname{Pr}\left(d \mid N^{\mathbf{t}^{\prime}}\right)
$$

for all $d \neq d^{*}$ and all $\mathbf{t}^{\prime}$ (including $\mathbf{t}$ ) defined as above. Thus, informative voting being efficient implies that (10) and (11) are equivalent. (In words, efficiency of the chosen decision at any type profile can only depend on the sets of voters exceeding the cut-points for $p$ and $q$.) 
(b) Suppose that

$$
\frac{\pi}{1-\pi}>\left(\frac{1-F_{0}(\hat{t})}{1-F_{1}(\hat{t})}\right)^{n^{\mathrm{t}}}\left(\frac{F_{0}(\hat{t})}{F_{1}(\hat{t})}\right)^{n-n^{\mathrm{t}}}
$$

for some profile $\mathbf{t}$. As $\left|N^{\mathbf{t}}\right|=n^{\mathbf{t}}$, this can be written as

$$
\pi\left(1-F_{1}(\hat{t})\right)^{\left|N^{\mathbf{t}}\right|} F_{1}(\hat{t})^{\left(n-\left|N^{\mathbf{t}}\right|\right)}>(1-\pi)\left(1-F_{0}(\hat{t})\right)^{\left|N^{\mathbf{t}}\right|} F_{0}(\hat{t})^{\left(n-\left|N^{\mathbf{t}}\right|\right)},
$$

which is equivalent to $\operatorname{Pr}\left(s_{1} \mid N^{\mathbf{t}}\right)>\operatorname{Pr}\left(s_{0} \mid N^{\mathbf{t}}\right)$. By part (a) of the proof, this is equivalent to $\operatorname{Pr}\left(s_{1} \mid \mathbf{t}\right)>\operatorname{Pr}\left(s_{0} \mid \mathbf{t}\right)$, which, in turn, implies

$$
\frac{\pi}{1-\pi}>\prod_{i \in\{1, \ldots, n\}} \frac{f_{0}\left(t_{i}\right)}{f_{1}\left(t_{i}\right)}
$$

by (7) and (8). Thus, we have proved that the right-hand side of (9) implies the left-hand side. By converting this argument the converse implication follows.

(c) For the converse implication of the theorem, assume that there is a type $\hat{t}$ which satisfies condition (2) for each type profile, and assume informative voting with $\hat{t}$ as cutpoint. Consider a voting rule $g$ defined as follows for every voting profile $\mathbf{v}$ :

$$
g(\mathbf{v})=1 \Longleftrightarrow \frac{\pi}{1-\pi}>\left(\frac{1-F_{0}(\hat{t})}{1-F_{1}(\hat{t})}\right)^{n^{\mathbf{v}}}\left(\frac{F_{0}(\hat{t})}{F_{1}(\hat{t})}\right)^{n-n^{\mathbf{v}}},
$$

where $n^{\mathbf{v}}$ is the number of 1 -votes in $\mathbf{v}$. We show that $g$ makes informative voting with $\hat{t}$ efficient. Consider type profile $\mathbf{t}$ and let $\mathbf{v}=\boldsymbol{\sigma}^{\hat{t}}(\mathbf{t})$. Suppose that $g(\mathbf{v})=1$. By (14) and $n^{\mathbf{v}}=n^{\mathrm{t}}$, we can write $\frac{\pi}{1-\pi}>\left(\frac{1-F_{0}(\hat{t})}{1-F_{1}(\hat{t})}\right)^{n^{\mathrm{t}}}\left(\frac{F_{0}(\hat{t})}{F_{1}(\hat{t})}\right)^{n-n^{\mathrm{t}}}$. By $(2)$, we have $\frac{\pi}{1-\pi}>$ $\prod_{i \in\{1, \ldots, n\}} \frac{f_{0}\left(t_{i}\right)}{f_{1}\left(t_{i}\right)}$. Hence, by $(7)$ and $(8), d=1$ is efficient for $\mathbf{t}$. The case where $g(\mathbf{v})=0$ can be shown similarly.

Proof of Theorem 3. Consider a voting rule $g: \mathcal{J}^{n} \rightarrow \mathcal{J}$ and suppose $g$ makes informative voting with some cut-point $\hat{t}$ efficient. By Theorem 2, $\hat{t}$ satisfies (2) for every type profile $\mathbf{t}$. We show that $g$ is the quota rule with threshold $m^{*}$.

Consider any type profile $\mathbf{t} \in[0,1]^{n}$ and let $\mathbf{v}=\boldsymbol{\sigma}^{\hat{t}}(\mathbf{t})$. Suppose $g(\mathbf{v})=1$. By informative voting being efficient, $d=1$ must be efficient; hence, $\operatorname{Pr}\left(s_{1} \mid \mathbf{t}\right)$ is maximal. From $\operatorname{Pr}\left(s_{1} \mid \mathbf{t}\right)>\operatorname{Pr}\left(s_{0} \mid \mathbf{t}\right)$, we have $\frac{\pi}{1-\pi}>\prod_{i \in\{1, \ldots, n\}} \frac{f_{0}\left(t_{i}\right)}{f_{1}\left(t_{i}\right)}$ by $(7)$ and (8). By Theorem 2 , this is equivalent to $\frac{\pi}{1-\pi}>\left(\frac{1-F_{0}(\hat{t})}{1-F_{1}(\hat{t})}\right)^{n^{\mathrm{t}}}\left(\frac{F_{0}(\hat{t})}{F_{1}(\hat{t})}\right)^{n-n^{\mathrm{t}}}$. If we denote the number of 1 -votes in $\mathbf{v}$ by $n^{\mathbf{v}}$, this expression can be written as $\frac{\pi}{1-\pi}>\left(\frac{1-F_{0}(\hat{t})}{1-F_{1}(\hat{t})}\right)^{n^{\mathbf{v}}}\left(\frac{F_{0}(\hat{t})}{F_{1}(\hat{t})}\right)^{n-n^{\mathbf{v}}}$ since $n^{\mathbf{v}}=n^{\mathbf{t}}$ under informative voting. Hence,

$$
g(\mathbf{v})=1 \Longleftrightarrow \frac{\pi}{1-\pi}>\left(\frac{1-F_{0}(\hat{t})}{1-F_{1}(\hat{t})}\right)^{n^{\mathbf{v}}}\left(\frac{F_{0}(\hat{t})}{F_{1}(\hat{t})}\right)^{n-n^{\mathbf{v}}},
$$

which, by (3) and by the right hand side of the inequality being decreasing in $n^{\mathbf{v}}$ implies

$$
g(\mathbf{v})=1 \Longleftrightarrow n^{\mathbf{v}} \geq m^{*}
$$




\subsection{Informative voting and best replies}

We have shown in the paper that informative voting is not always efficient but if it is, a quota rule has to be used. Here, we argue that if a quota rule is used and each voter plays a best reply, then we may assume informative voting. Let $n^{\mathbf{v}}$ denote the number of 1 -votes in a given voting profile $\mathbf{v}$. Recall that a voting rule $g$ is a quota rule if there is a $k \in\{0, \ldots, n+1\}$ such that

$$
g(\mathbf{v})= \begin{cases}0 & \text { if } n^{\mathbf{v}}<k \\ 1 & \text { if } n^{\mathbf{v}} \geq k\end{cases}
$$

Let $g$ be a quota rule, $i \in\{1, \ldots, n\}$, and let $\sigma_{i}:[0,1] \rightarrow\{0,1\}$ be a best reply of voter $i$ against a strategy profile $\boldsymbol{\sigma}_{-i}$ of the other voters in the Bayesian game induced by $g$. Suppose $\sigma_{i}\left(t_{i}\right)=1$ for some $t_{i} \in[0,1)$, so that

$$
E\left(u\left(g\left(1, \boldsymbol{\sigma}_{-i}\left(\mathbf{t}_{-i}\right)\right), S \mid t_{i}\right) \geq E\left(u\left(g\left(0, \boldsymbol{\sigma}_{-i}\left(\mathbf{t}_{-i}\right)\right), S \mid t_{i}\right),\right.\right.
$$

where $\mathbf{t}_{i}$ is a type profile of the other voters and $\left.\sigma_{i}\left(\mathbf{t}_{-i}\right)\right)$ the vector of associated actions. Consider $t_{i}^{\prime}>t_{i}$. Then, we must have

$$
E\left(u\left(g\left(1, \boldsymbol{\sigma}_{-i}\left(\mathbf{t}_{-i}\right)\right), S \mid t_{i}^{\prime}\right) \geq E\left(u\left(g\left(0, \boldsymbol{\sigma}_{-i}\left(\mathbf{t}_{-i}\right)\right), S \mid t_{i}^{\prime}\right) .\right.\right.
$$

To prove (16), since $g$ is a quota rule, we only have to consider the case where $g\left(1, \boldsymbol{\sigma}_{-i}\left(\mathbf{t}_{-i}\right)\right)$ $=1$ and $g\left(0, \boldsymbol{\sigma}_{-i}\left(\mathbf{t}_{-i}\right)\right)=0$; i.e., voter $i$ is pivotal. Note that (15) implies that $\operatorname{Pr}(S=$ $\left.s_{1} \mid t_{i}\right) \geq \operatorname{Pr}\left(S=s_{0} \mid t_{i}\right)$, given the strategies of the other voters. Since $t_{i}^{\prime}>t_{i}$, it is easy to derive that then also $\operatorname{Pr}\left(S=s_{1} \mid t_{i}^{\prime}\right) \geq \operatorname{Pr}\left(S=s_{0} \mid t_{i}^{\prime}\right)$, which in turn implies (16).

Hence, we may assume that $\sigma\left(t^{\prime}\right)=1$. If we let $\hat{t}$ be the infimum of the set $\{t \in(0,1)$ : $\sigma(t)=1\}$ - assuming that this infimum is in $(0,1)$ - then we may assume that voter $i$ votes informatively with cut-point $\hat{t}$.

\section{References}

Austen-Smith, D., Banks, J. (1996) Information aggregation, rationality, and the Condorcet jury theorem. American Political Science Review 90: 34-45

Austen-Smith, D., Feddersen, T. (2006) Deliberation, preference uncertainty, and voting rules. American Political Science Review 100(2), 209-217

Azrieli, Y., Kim, S. (2014) Pareto efficiency and weighted majority rules. International Economic Review 55(4): 1067-1088.

Barelli, P., Bhattacharya, S., Siga, L. (2017). On the possibility of information aggregation in large elections. Working paper

Bozbay, I., Dietrich, F., Peters, H. (2014) Judgment aggregation in search for the truth. Games and Economic Behavior 87: 571-590

Coughlan, P. J. (2000). In defense of unanimous jury verdicts: Mistrials, communication, and strategic voting. American Political Science Review 94(2), 375-393. 
Duggan, J., Martinelli, C. (2001) A Bayesian model of voting in juries. Games and Economic Behavior 37(2): 259-294

Feddersen, T., Pesendorfer, W. (1997) Voting behavior and information aggregation in elections with private information. Econometrica 65(5): 1029-1058

McLennan, A. (1998) Consequences of the Condorcet Jury Theorem for beneficial information aggregation by rational agents. American Political Science Review 92 (2): $413-418$

Meirowitz, A. (2002) Informative voting and Condorcet jury theorems with a continuum of types. Social Choice and Welfare 19: 219-236

Schmitz, P. W., Tröger, T. (2012). The (sub-) optimality of the majority rule. Games and Economic Behavior 74(2): 651-665. 